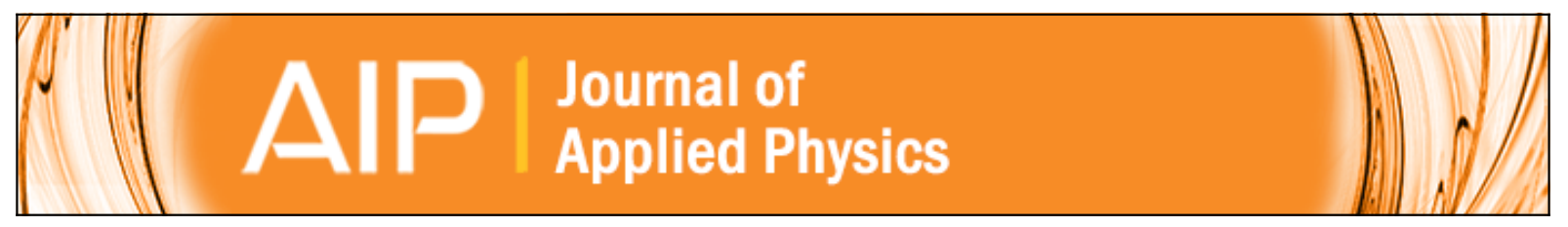

\title{
Resonant band-f scattering and the magnetic properties of highly correlated actinide systems
}

G.-J. Hu, N. Kioussis, B. R. Cooper, and A. Banerjea

Citation: Journal of Applied Physics 61, 3385 (1987); doi: 10.1063/1.338780

View online: http://dx.doi.org/10.1063/1.338780

View Table of Contents: http://scitation.aip.org/content/aip/journal/jap/61/8?ver=pdfcov

Published by the AIP Publishing

\section{Articles you may be interested in}

Electronic and magnetic properties of manganite thin films with different compositions and its correlation with transport properties: An X-ray resonant magnetic scattering study

J. Appl. Phys. 116, 222205 (2014); 10.1063/1.4902959

$5 \mathrm{f}$ band dispersion in the highly correlated electronic structure of uranium compounds

AIP Conf. Proc. 532, 420 (2000); 10.1063/1.1292353

A high repetition rate solid state laser system for resonance ionization mass spectrometry of actinides AIP Conf. Proc. 454, 285 (1998); 10.1063/1.57161

Correlated band magnetism of cerium and actinide materials

J. Appl. Phys. 81, 3856 (1997); 10.1063/1.364732

Stabilization of the $5 f$ Energy Band in Actinide- Rh 3 Intermetallic Compounds

AIP Conf. Proc. 10, 1076 (1973); 10.1063/1.2946747

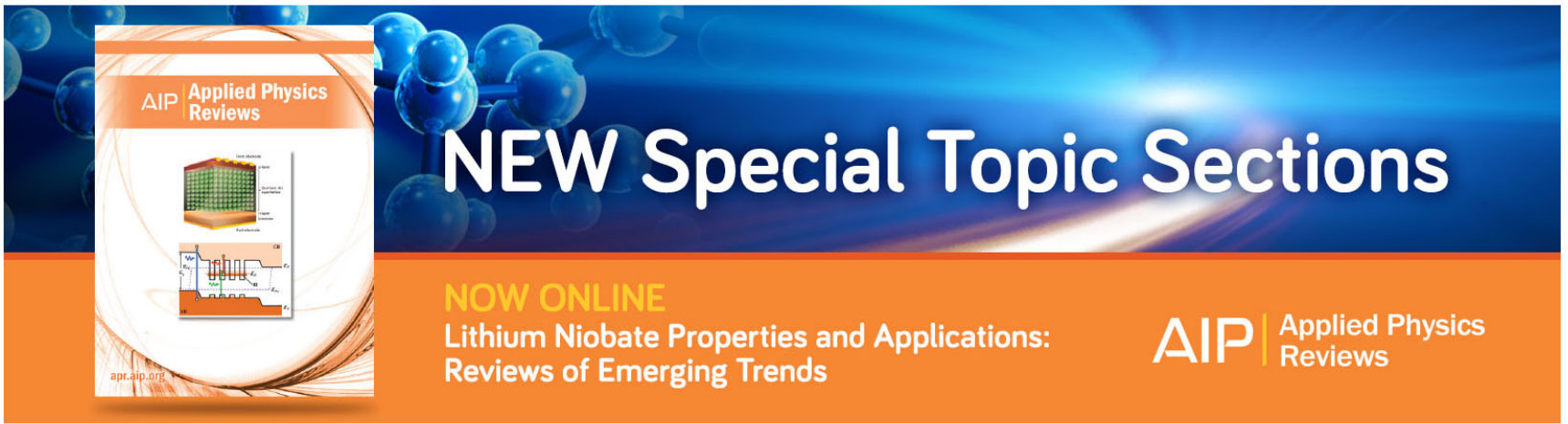




\title{
Resonant band $f$ scattering and the magnetic propertles of highly correlated actinide systems
}

\author{
G.-S. His, N. Kloussis, and B. R. Cooper \\ Department of Physics, West Virginia University, Morganiown, West Virginia 26506
}

A. Baneriea

Department of Physics, West Virginia University, Morgantown, West Virginia 26506 and General Motors

Research Laboratory, "W Wren, Michigan 48090

To understand the magnetic properties of moderately delocalized light actinide (uranium, nepunium, plutonium) systems, it is necessary to treat correctly the way in which the highy correlated behavior of the $f$ electrons within the actinide ion is linked to the non $f$ band behavior via the hybridization process. We do this by transforming the hybridization into band-f resonant scattering. We have successfully applied the theory to PuSb by considering both dominant and next-to-dominant scattering channels.

\section{MTRODUCTION}

The magnetic behavior of the cerium monoprictides has been understood ${ }^{2}$ on the basis of interactions that arise from the hybridization of non-f band electrons with moderately delocalized $f$ electrons. This hybridization was first treated by Coqbin and Schrieffer ${ }^{2}$ by transforming it into band $f$ resonant scattering from $\mathrm{Ce}^{3+}\left(f^{3}\right)$ ions. Extending this theory to the general case of $f$ ions with more than one $f$ electron in each ion, Cooper et al. ${ }^{1,3-5}$ reported the results of calculations for the $\mathrm{Pu} u^{3+}\left(f^{5}\right)$ system in the cases of $j j$ coupling, $\mathrm{L}$ $\$$ coupling, and intermediate coupling. Success was achieved ${ }^{5}$ in explaining the main features ${ }^{4,6}$ of the anisotropic magnetic equilibrium behavior of $\mathrm{PuSb}$, namely, the variation of the magnetization with temperature and phase trarsition from a ferromagnetic phase to a long-period antiferromagnetic phase; but the theory failed to reproduce the correct polarization (longitudinal to the modulation direction) for the long-period antiferromagnetic structure observed in the temperature range below the Neel tempera. ture $T_{N}$. The theory also gave ${ }^{7}$ a susceptibility singularity in the near-critical region corresponding to the incorrect polarization. For the excitation behavior, the theory failed to explain why for PuSb there are ${ }^{8}$ two modes at the zone boundary for the wave vector perpendicular to the magnetic moment. (These modes are at least partially polarized, parallel and perpendicular to the wave vector, respectively. ${ }^{8}$ )

\section{RESONANT SCATERRING THEORY ANO RESULTS}

In the original calculations ${ }^{2-12}$ for $\mathrm{Ce}^{3+}$ systems, the dominant contribution to the lowest order in $\left(k_{F} R\right)^{-1}$ to the two-ion interaction came from processes in which the electron involved in the associated single-site scattering event had magnetic quantum number (with respect to the interionic axis) $m_{j}= \pm 1 / 2\left(m_{l}=0, m_{s}= \pm 1 / 2\right)$; however, some contribution from $m_{j}= \pm 1 / 2$ states with $m_{l}=+1$ was also included. On extending the theory to systems with more than one $f$ electron per ion, Thayamballi and Cooper ${ }^{3}$ and Banerjea et al. ${ }^{5}$ assumed that oniy one electron (out of the many-electron ionic state) with $m_{l}=0$

\footnotetext{
a) Present address.
}

$\left(m_{j}= \pm 1 / 2\right)$ (one-electron component that points its charge density along the interionic axis taken as the axis of quantization) is involved in the scattering events and the quantum numbers of all the other one-electron components remain unchanged. To remove the discrepancies between theory and experiment we recognized that the $m_{j}= \pm 1 / 2$ states with $m_{i}= \pm 1$, partially included for the $\mathrm{Ce}^{3+}$ theory, must play a crucial "fine tuning" role. (These one-electron states "point" their charge densities of the interionic axis.) Therefore, we have now considered contributions to the two-ion interaction from the following three single-site scattering channels:

(1) $m_{l}=0, \quad m_{s}= \pm 1 / 2 \leftrightarrow m_{l}^{l}=0, \quad m_{s}^{\prime}= \pm 1 / 2$;

(2) $m_{i}=0, \quad m_{s}= \pm 1 / 2 \leftrightarrow m_{i}^{\prime}= \pm 1, \quad m_{s}^{\prime}=\mp i / 2$;

(3) $m_{l}= \pm 1, m_{s}=\mp 1 / 2 \leftrightarrow m_{1}^{\prime}= \pm 1, m_{s}^{\prime}=\mp 1 / 2$.

In the earlier studies ${ }^{3,5}$ the only channel considered was (1), which, in orders of $\left(k_{F} R\right)^{-1}$, is the leading contribution to the two-ion interaction. After including the next two higherorder contributions, arising from channels 2 and 3 , in our calculation, we have obtained some striking results.

\section{A. Longitudinal polarzation}

Figure 1 shows the polarization diagram in the $W_{1}$ vs $W_{2}$ plane, i.e. shows whether the long-period antiferromagnetic phase has transverse or iongitudinal polarization. Here the phenomenologically introduced weighting factor $W_{1}$ $\left(W_{2}\right)$ measures the ratio of the magnitude of contribution to the strength of two-ion interaction (range parameters) of channel $2(3)$ to that of channel 1 . For the L-S coupling case we find that for a wide range of values of $W,(0.38-1.0)$ or of $W_{2}(0.19-1.0)$ the magnetic behavior of the $\mathrm{Pu}^{3+}$ system, corresponding to $\mathrm{PuSb}$, predicted by the theory gives a longitudinally polarized long-period antiferromagnetic phase. However, in the area with $W_{1}<0.38$ and $W_{2}<0.19$ the polarization is transverse. On the critical line, which separates the longitudinal and transverse areas, the antiferromagnetic phase is unpolarized. It is clear to see that at the point $W_{1}=0, W_{2}=0$, which is the case treated in the previous studies, ${ }^{3-5}$ the polarization is transverse. Thus the contribu- 


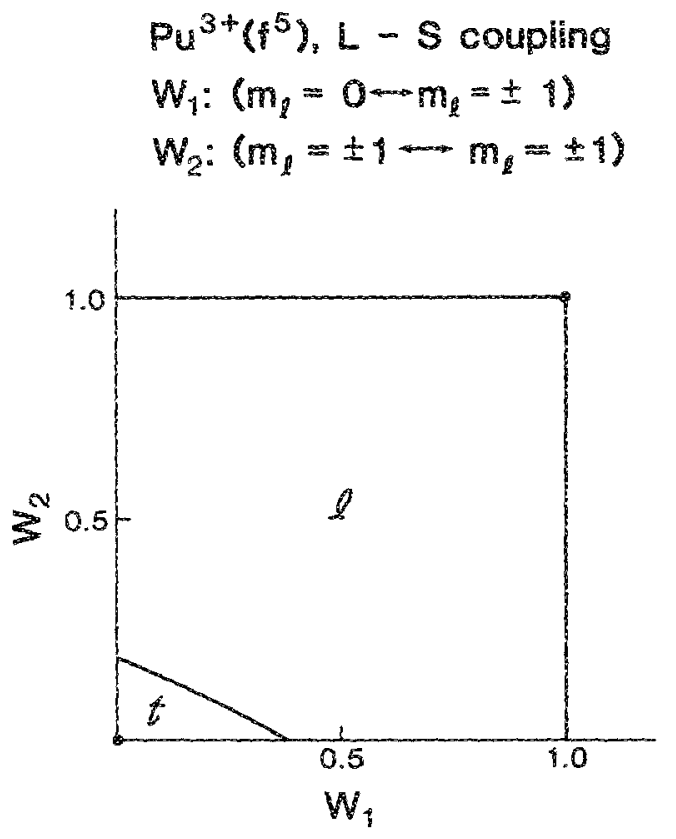

FIG. 1. Polarization behavior as a function of the weighting factors $W_{1}$ and $W_{2}$ for $\mathrm{Pu}^{3+}\left(f^{5}\right)$ system in the antiferromagnetic phase (in the temperature range below Néel temperature $T_{N}$ ). The longitudinal and transverse polarized phases are labeled by $l$ and $i$, respectively.

tion of the channels with $m_{l}= \pm 1$ "tunes" the interaction to provide the experimentally observed polarization in $\mathrm{PuSb}$.

\section{Corration length anisotropy}

The ratio $\kappa_{\|} / \kappa_{1}$ of the inverse critical correlation lengths parallel and perpendicular to moment direction, evaluated at $T-T_{N}=0.04 T_{N}$, with negative crystal-field splitting $\left(\Gamma_{8}\right.$ low) has been calculated for L-S coupling for the case of a longitudinally polarized antiferromagnetic structure with $W_{1}=1, W_{2}=0, E_{2}=E_{1}, E_{3}=-0.28 E_{1}$. These parameters provide equilibrium behavior for $\mathrm{PuSb}$ closely approximating the experimental behavior. We find that $\kappa_{1} / \kappa_{1} \approx 2.2$, and this value is almost independent of the crystal field. The value of $\kappa_{11} / \kappa_{1}$ is greater than unity, indicating that the magnetic interactions are stronger within the ferromagnetic planes than are the interplanar couplings. This value of $\kappa_{1 /} / \kappa_{1}$ is in close agreement with that of very recent experiments ${ }^{13}$ giving $\kappa_{1} / \kappa_{1}=1.8 \pm 0.2$ for PuSb. Thus we note two important changes from the previous results ${ }^{7}$ that did not include any contribution from scattering channels (2) and (3) (the $m_{l}= \pm 1$ channeis). The singularity in the near-critical susceptibility now provides the correct polarization as experimentally observed; and the direction of anisotropy for $\mathrm{L}-\mathrm{S}$ coupling is reversed from that previously found $\left(\kappa_{\|} / \kappa_{1}>1\right.$ as has now been experimentally observed ${ }^{13}$ ).

\section{Two elliptically polarized modes in excitation spectrum}

In neutron inelastic scattering experiments Lander et $a l .^{8}$ recently found a number of interesting features in the excitation spectrum of PuSb in the low-temperature phase. Particularly interesting is that there exist two modes, on ap- proaching the zone boundary in the transverse direction [100] (the modulation is along [001] direction), and these modes are at least partially linearly polarized (i.e., are at least elliptically rather than circularly polarized). At the zone boundary the mode at $4.3 \mathrm{THz}$ has $y$ polarization (transverse to wave vector) whereas the mode at $3.5 \pm 0.2$ THz has $x$ polarization (parallel to wave vector). At $(0.7,0,0)$ two separate modes can no longer be distinguished.

The present theory allows us to understand this unusual feature. Figure 2 shows the calculated excitation spectra at $T=0 \mathrm{~K}$, for the $\mathrm{L}-\$$ coupling case with $W_{1}=1, W_{2}=0$, $E_{2}=E_{1}, \quad E_{3}=-0.306 E_{1}, \quad 60 B_{4}=-0.38 E_{1} \quad\left(E_{1}\right.$ $=121.43 \mathrm{~K}$ was chosen to match the experimental Neel temperature, $T_{N}=85 \mathrm{~K}$ ). Here $E_{1}, E_{2}, E_{3}$ are the range parameters ${ }^{1}$ and $B_{4}$ is the crystal-field parameter. The crystal-field splitting $\Delta_{C F}$, which is strongly modified ${ }^{14}$ by the hybridization, is treated phenomenologically with $\Delta_{\mathrm{CF}}=360 B_{4} ;$ and a negative $B_{4}$ gives a $\Gamma_{8}$ ground state. The solid lines in Fig. 2 show the intense modes which are likely to be observed experimentally. The dominant mode $L_{41}$ has an excitation energy of about $3.4 \mathrm{THz}(164 \mathrm{~K})$ at the $\Gamma$ point and increases in energy as we go toward the $X$ points. At about $(0.7,0,0)$ the mixing of the transverse modes $L_{31}$ and $L_{41}$ starts to occur. This is associated with the reduction of the intensity of the $L_{41}$ mode, with a corresponding increase in the $L_{31}$ mode intensity. This admixture produces two ei-

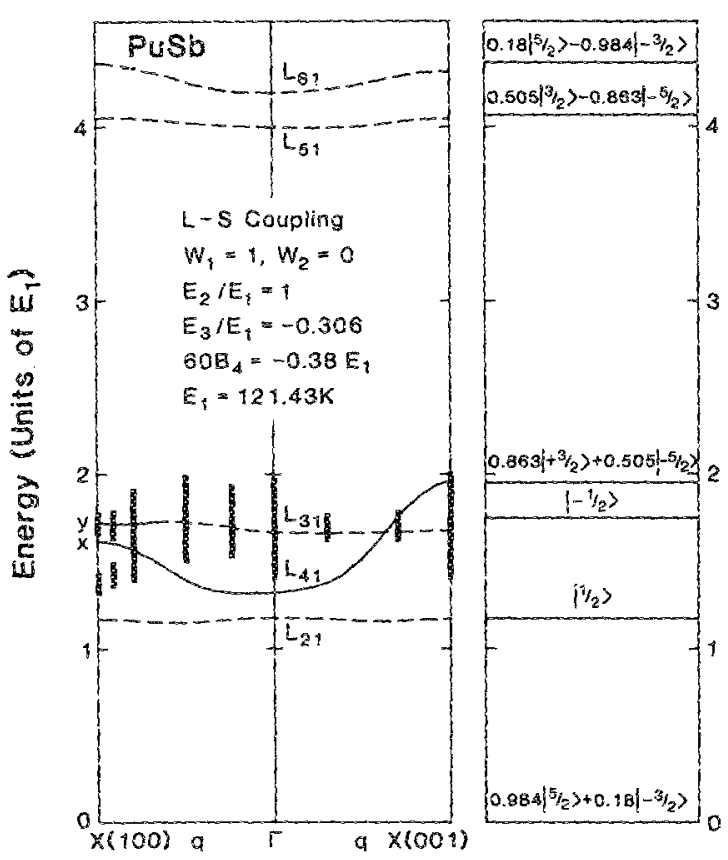

FIG. 2. Dispersion curves for excitations in the ferromagnetic phase at $T=0$ with L-S coupling, $E_{2}=E_{1}, E_{3}=0.306 E_{1}$, and $B_{4}=-(0.38)$ 60) $E_{1}$ with weighting $W_{1}=1, W_{2}=0$ for along the $[100\}$ and $[001]$ (directions parallel and perpendicular to the moment direction, respective(y). $E_{1}=121 \mathrm{~K}$ to match experimental $T_{N}$. The solid curves $L_{31}$ near $X(100)$ and $L_{41}$ show the most intense mode. Modes $L_{31}, L_{41}$, and $L_{51}$ are transverse modes and modes $L_{61}$ and $L_{21}$ are longitudinal and quadrupolar modes, respectively. The energy levels of the molecular-field states and their compositions in terms of the angular momentum eigenstates (quantized along [001]) are shown on the far right. Heavy bars show the experimental results of Lander et $a t$. (Ref. 8) for PuSb. 
liptically polarized branches: a y-polarized (major axis) upper branch and an $x$-polarized lower branch. At the zone boundary $[q=(1,0,0)$ direction $]$ the wo predicted modes have energies $4.5 \mathrm{THz}(216 \mathrm{~K})$ and $4 \mathrm{THz}(194 \mathrm{~K})$, respectively, and the polarization $\left(\left\langle J_{y}^{2}\right\rangle /\left\langle J_{x}^{2}\right\rangle\right)^{1 / 2}$ is $\sqrt{2}$ for the upper branch and $(1 / \sqrt{6})$ for the lower branch.

The relative separations between modes are quite sensitively dependent on the value of $B_{4}$. To bring modes $L_{31}$ and $L_{41}$ close enough to cause the admixture at the zone boundary (i.e., to give two intense modes) the value of $60 B_{4}$ must be chosen in a narrow range from $-0.35 E_{1}$ to $-0.40 E_{1}$. Outside this range of $B_{4}$ the only intense mode is $L_{41}$, and this is circulariy polarized. In fact, we chose $B_{4}$ to provide the mode mixing giving two intense (and elliptically polarized) modes at the zone boundary. It is a remarkable result that this choice of crystal-field spitting parameter places the mode energies almost exactly at the experimental values. This result indicates that for $\mathrm{PuSb}$ the crystal-neld splitting from $\Gamma_{g}$ to $\Gamma_{7}$ should be about $255-290 K$. Further experimental information checking this predicted value would be valuable.

We have also carried out calculations for the $\mathrm{Pu}^{3+}$ system in the case of intermediate coupling (IC). After a te. dious evaluation we found almost the same results as we have obtained in the $L-S$ coupling limit. This is not surprising since the ground state of the L-S coupling limit the predominant component of the $1 \mathrm{C}$ ground state which we take as $75 \%{ }^{6} H$ and $25 \%{ }^{4} \mathrm{G}$.

One discrepancy exists between the calculated and observed excitation behavior at low temperature. While the experimentally observed magnetic transition is almost dis. persionless for along the [001] direction, and a clearly defined minimum occurs for the low-energy branch at the zone boundary ( $X$ point), our theory predicts that the intense mode has significant dispersion for along both the [001] and [100] directions with an energy minimum at the $\Gamma$ point regardless of the choice of phenomenological param eters.

In conciusion, we have found that upon including the additional (next-to-dominant) channels for the single-site scattering events the inybridization-mediated interaction fulIy explains the anisotropic magnetic equilibrium behavior for the $\mathrm{Pu}^{3+}\left(f^{5}\right)$ system. The longitudinally polarized antiferromagnetic phase has been satisfactorily obtained by considering the contribution of the additional scattering channels, which is equivalent to including off-axis felectron charge distribution. We also obtain excellent agreement with experiment ${ }^{13}$ for the correlation length anisotropy. The theory also gives a magnetic excitation spectrum at $T=0$ in the ferromagnetic phase with two polarized branches at the zone boundary with along the [100] (transverse to moment) direction. The predicted crystal-field splitting from $\Gamma_{8}$ to $\Gamma_{7}$ is about $255-290 \mathrm{~K}$.

\section{ACKNOWEDGEST}

This research has been supported by the U.S. Department of Energy through Grant DE-FGO5-84-ER45134.

13. R. Cooper, R. Siemanzr, D. Yang, P. Thayamballi, and A. Banerjea, Handbook on the Physics and Chemistry of the Actinides, edited by A. I. Freeman and G. H. Lander (North-Holland, Amsterdan, 1985), Vol. 2, Chap. 6, pp. 435-500.

${ }^{2}$ B. Cogblin and J. R. Schrieffer, Phys. Rev. 285, 847 (1969).

${ }^{3}$ P. Thayamballi and B. R. Cooper, J. Appl. Phys. 55, 1829 (1984).

${ }^{4}$ B. R. Cooper, P. ThayambalH, J.C. Spirlet, W. Muller, and O. Yogt, Phys. Rev. Lett. 5R, 2418 (1985).

${ }^{5}$ A. Banerjea, B. R. Cooper, and P. Thayamballi, Phys, Rev. B 30, 2671 (1984); A. Ganerjea and B. R. Cooper, Phys. Rev. B 34, 1607 (1986).

D. Burlet, S. Quezel, J. Rossat-Mignod, J. C. Spiriet, J. Rebizant, W. Müller, and O. Vogt, Rhys. Rev. B 30, 6660 (1984).

${ }^{7}$ N. Kioussis and B. R. Cooper, Phys. Rev. B 34, 3261 (1986),

${ }^{8}$ G. H. Lander, W. G. Stirling, J. Rossat-Mignod, J. C. Spirlet, I. Rebizant, and $O$. Vogt, Physica 368 , 409 (1986).

'R. Siemann and B. R. Cooper, Phys. Rev. Lett. 44, 1015 (1980).

10. R. Cooper, J. Magn. Magn. Mater. 29, 230 (1982).

"D. Yang and B. R. Cooper, J. Appl. Phys. 53, 1988 (1982).

${ }^{12}$ B. R. Cooper, I. Thayambsili, and D. Yang, I. Appl. Phys. 55, 1866 (1984); P. Thayamballi, D. Yang, and B. R. Cooper, Phys. Rev. B 29, $\$ 049$ (1984).

${ }^{13}$ P. Burlet, J. Rossat-Mignod, G. H. Lander, J. C. Spirlet, J. Rebizant, and O. Vogt, Phys. Rev. B (to be published).

${ }^{14}$ J. M. Wills, B. R. Cooper, and P. Thayambsilt, J. Appt. Phys. 57, 3183 (1985). 